\title{
Advanced Synovial Sarcoma
}

National Cancer Institute

\section{Source}

National Cancer Institute. Advanced Synovial Sarcoma. NCI Thesaurus. Code C153184.

A synovial sarcoma that has spread extensively to other anatomic sites or is no longer responding to treatment. 